\title{
High-speed AFM and nano-visualization of biomolecular processes
}

\author{
Toshio Ando • Takayuki Uchihashi • Noriyuki Kodera • \\ Daisuke Yamamoto • Atsushi Miyagi • \\ Masaaki Taniguchi • Hayato Yamashita
}

Received: 24 September 2007 / Accepted: 15 November 2007 / Published online: 20 December 2007

(C) Springer-Verlag 2007

\begin{abstract}
Conventional atomic force microscopes (AFMs) take at least $30-60 \mathrm{~s}$ to capture an image, while dynamic biomolecular processes occur on a millisecond timescale or less. To narrow this large difference in timescale, various studies have been carried out in the past decade. These efforts have led to a maximum imaging rate of $30-60 \mathrm{~ms} /$ frame for a scan range of $\sim 250 \mathrm{~nm}$, with a weak tip-sample interaction force being maintained. Recent imaging studies using high-speed AFM with this capacity have shown that this new microscope can provide straightforward and prompt answers to how and what structural changes progress while individual biomolecules are at work. This article first compares high-speed AFM with its competitor (single-molecule fluorescence microscopy) on various aspects and then describes high-speed AFM instrumentation and imaging studies on biomolecular processes. The article concludes by discussing the future prospects of this cutting-edge microscopy.
\end{abstract}

Keywords Microscopy · Imaging · ATP hydrolysis . Motion $\cdot$ Kinetics

T. Ando $(\varangle) \cdot$ T. Uchihashi $\cdot$ N. Kodera $\cdot$ D. Yamamoto $\cdot$

A. Miyagi $\cdot$ M. Taniguchi $\cdot H$. Yamashita

Department of Physics, Kanazawa University,

Kakuma-machi,

Kanazawa 920-1192, Japan

e-mail: tando@kenroku.kanazawa-u.ac.jp

T. Ando $\cdot$ T. Uchihashi $\cdot$ N. Kodera $\cdot$ D. Yamamoto

CREST/JST,

Sanban-cho, Chiyoda-ku,

Tokyo 102-0075, Japan

\section{Introduction}

Protein molecules dynamically change their structure during functioning. Therefore, their functional mechanisms cannot be understood only from the ensemble average information such as crystal structures at different static states and biochemical properties. We need single-molecule techniques that will allow us to track and measure individual biomolecules at work. The recent developments in fluorescence video microscopy allow us to observe the translational or rotational motion of the individual fluorescent spots that are emitted from fluorophores attached to biomolecules. Such observations are valuable for dissecting the dynamic behavior of the labeled biomolecules in action [1-3]. However, we still need to infer from the observed behavior of the fluorescent spots how the labeled molecules actually behave. For this, if possible, we should directly observe single molecules themselves at nanometer spatial resolution and millisecond temporal resolution. This would be a more straightforward approach to understanding biomolecular behavior than observing fluorescent spots.

Unlike optical microscopy, AFM can directly visualize unstained biomolecules in physiological solutions at nanometer resolution. This unique capability inspired researchers to apply it to the observation of biomolecular processes. For example, in 1989, Paul Hansma and his colleagues observed at $\sim 1$-min intervals the fibrin clotting process which was initiated by the digestion of fibrinogen with thrombin [4]. Using tapping mode AFM [5], Hansma, Bustamante, and their colleagues obtained time-lapse images ( $\sim 30$-s intervals) of the RNA replication reaction by DNA and RNA polymerase [6] and of the onedimensional diffusion of RNA polymerase along a DNA strand [7]. However, as biomolecular processes generally occur on a millisecond timescale or less, the image 
acquisition time ( $30 \mathrm{~s}$ to a few minutes) was too long to capture images in real time.

Attempts to increase the AFM imaging rate were initiated in 1991 by Quate and his colleagues. Their attempts are made with the aim of fast inspection or lithography of a wide range of surfaces of hard materials [8], rather than of observing biomolecular processes. Images (a few micrometer scan range) of a metalsemiconductor field-effect transistor were taken at a frame rate of $\sim 0.3 \mathrm{~s} /$ frame in constant-height mode (i.e., no feedback scan in the $z$-direction). This study proceeded towards the development of cantilevers with integrated sensors and/or actuators $[9,10]$ and cantilever arrays with self-sensing and self-actuation capabilities [11-13]. Unfortunately, fabrication of these cantilevers could be made only in relatively large dimensions, resulting in low resonant frequencies (although higher than those of conventional tube scanners) and large spring constants. Insulation coating of the integrated cantilevers for the use in liquids further lowered the resonant frequency [14]. Thus, this approach has not come to fruition of realizing high-speed AFM for biological research.

Studies aiming at realizing high-speed AFM for biological research were pioneered around 1994. Some results from these studies gradually began making an appearance during 1996-2002. In 1998, a fast-imaging study was reported [15], in which an image of DNA was taken in $1.7 \mathrm{~s}$ using small cantilevers and an optical deflection detector [16] designed for them. A more complete high-speed AFM system was reported by us in 2001 [17] and 2002 [18], wherein a high-speed scanner and fast electronics were introduced in addition to small cantilevers (resonant frequencies $\sim 600 \mathrm{kHz}$ in water) and an optical deflection detector for them. An imaging rate of $80 \mathrm{~ms} /$ frame was achieved, and therefore, moving myosin $\mathrm{V}$ molecules were filmed in successive images. This stage is considered as the first stage of high-speed bioAFM development.

In the second stage (2003-2007), the capability of highspeed AFM was further enhanced by the introduction of new devices such as active damping techniques for minimizing vibrations of the $z$-scanner [19] and a new feedback controller that could maintain a low tip-sample interaction force without sacrificing the scan rate [20]. Various imaging studies were attempted to capture biological processes on video. Some of these studies demonstrated the usefulness of high-speed AFM for elucidating the molecular mechanisms of proteins $[21,22]$. Now, the most advanced tapping-mode high-speed AFM can capture images on video at $\sim 30-60 \mathrm{~ms} /$ frame for a scan range of $\sim 250 \mathrm{~nm}$ under feedback operation capable of preventing weak proteinprotein interactions from being disturbed by the scanning tip $[23,24]$.
High-speed AFM is becoming a truly useful tool for biological sciences. We expect that in the near future, it will be fully established and commercially available, leading to a rapid increase in the user population. This article aims to provide potential users and developers with a concise introduction to high-speed AFM with regard to its features relative to single-molecule optical microscopy, instrumentation, application to biological studies, current capabilities and limitations, and future prospects. AFM movies have been placed at http:// www.s.kanazawa-u.ac.jp/phys/biophys/roadmap.htm.

\section{Comparison with TIRFM}

Total internal reflection fluorescence microscopy (TIRFM; [1-3]) is a single-molecule technique for tracking dynamic biomolecular processes that competes with high-speed AFM. It therefore seems worthwhile to compare these two microscopic techniques, as they are complementary in nature and have advantages and disadvantages over each other.

\section{Spatial resolution}

The greatest advantage of AFM is, of course, its high spatial resolution. The spatial resolution of optical microscopy is given by Abbe's law: $d=0.5 \lambda / \mathrm{NA}$, where $\lambda=$ wavelength of monochromic light and NA=limiting numerical aperture of an objective. The spatial resolution of AFM is approximately given by $\sqrt{2 r_{t}(\Delta z)_{\min }}$, where $r_{t}=$ radius of curvature of the tip apex and $(\Delta z)_{\min }=$ resolution limit of the sample height detection. For typical values of $r_{t}=$ $5 \mathrm{~nm}$ and $(\Delta z)_{\min }=0.3 \mathrm{~nm}$ with bioAFM, the spatial resolution becomes $\sim 2 \mathrm{~nm}$, which is $\sim 100$ times higher than that with conventional fluorescence microscopy. This resolution is still approximately ten times higher than a more recently developed fluorescence microscopy, namely, stimulated-emission-depletion (STED) fluorescence microscopy $[25,26]$. However, this high resolution of AFM is not sufficient to detect the binding of very tiny ligands (such as ATP) to a protein. In TIRFM, we can monitor the binding events using fluorescent ligands [27].

\section{Time resolution}

Fluorescence video microscopy is usually performed at an imaging rate of $33 \mathrm{~ms} /$ frame. This is comparable to the most advanced high-speed AFM, although the imaging area of optical microscopy is much wider than that of AFM. Fluorescence video microscopy can be performed at faster imaging rates, using bright fluorescent labels and faster video cameras. The imaging rate of AFM can be enhanced to $1 \mathrm{~ms} /$ frame [28]. This is accomplished by discarding the 
tapping-mode operation and the feedback control for maintaining a constant tip-sample interaction force. Without feedback control, it would be impossible to observe biomolecular processes.

\section{Noninvasiveness}

With regard to noninvasiveness to samples, optical microscopy has the strongest advantage, as long as illumination wavelengths longer than $\sim 500 \mathrm{~nm}$ are used. In high-speed tapping mode AFM, the tip-sample interaction force cannot be reduced to a negligible level for samples that contain delicate biomolecular interactions. Presently, a truly noncontact condition is realized only with in-vacuum AFM [29]. High-speed noncontact AFM (nc-AFM) for in-liquid samples will therefore become an important target in the near future.

\section{Floating molecules}

The advantage of AFM is that floating molecules in the solution are completely invisible, and therefore, their concentrations can be increased. When we study the dynamic interaction of a floating protein with the counterpart protein attached to the substratum, invisibility of floating molecules is quite advantageous. In TIRFM, the concentration of floating molecules with fluorophores cannot be increased beyond $50 \sim 100 \mathrm{nM}$, as an elevation in the concentration results in too much background fluorescence [27].

\section{Visibility}

With AFM, every object attached to a substratum and the substratum surface itself are visible (i.e., nonselective visibility). This feature is in marked contrast to TIRFM. With TIRFM, only fluorescently labeled molecules are visible, a feature that appears advantageous, as we can extract only what we would like to see. However, we always have to bear in mind that key information may be missed in the selective visualization. In addition, with TIRFM, photobleaching imposes serious limitations not only on the length of successive imaging but also on the measurement of slow kinetic association and dissociation events (i.e., difficulty in distinguishing the dissociation events from photobleaching; [30]). With AFM, it is sometimes hard to identify observed molecules contained in a multicomponent system; with TIRFM, however, this can be easily achieved by specific labeling with fluorophores or by GFP techniques. A recently developed technique, "recognition AFM" [31,32], appears to solve this problem. However, it has not been experimentally tested whether recognition imaging is compatible with high-speed imaging. This issue will be discussed later. Due to the nonselective visibility with AFM, the substratum surface should have very small roughness. This condition narrows the range of our choice for a substratum.

\section{Combined optical microscope and high-speed AFM}

As described above, the two microscopic techniques have complementary features. For single-molecule observations of biomolecular processes, combining these techniques into a single instrument would therefore be useful. For example, we can visualize dynamic protein structural changes by high-speed AFM while recording simultaneously optical signals of chemical reactions such as ATP hydrolysis at individual protein molecules. Macromolecules such as protein, DNA, or complexes such as chromosomes are stretched by optical tweezers while their structural changes are being imaged by AFM. Chemical specification of an object imaged by AFM can be made from its Raman or fluorescence signals. There have been a number of reports on conventional slow AFM combined with confocal fluorescence [33-36] or conventional Raman scattering [37, 38] microscopes, coherent anti-Stokes Raman scattering [39, 40] microscopes, or TIRFM [41-43]. When still images are taken, simultaneous recording of optical and AFM images is not necessarily required. When simultaneous recording is required, various precautions are necessary. Cantilever tips of amorphous silicon and amorphous silicon nitride are known to be strongly photoluminescent [44-46], while crystalline silicon tips are much less luminescent [33]. EBD carbon tips are also less luminescent. As silicon nitride tips are usually amorphous, and therefore, its luminescence has to be filtered out, which narrows our choice of wavelengths used in optical microscopy. We need to use crystalline silicon tips or EBD carbon tips particularly in multicolor confocal imaging. UV, near UV and visible lights are absorbed in this order by gold often used for cantilever coating. The thermal expansion coefficient of gold is larger than those of silicon and silicon nitride, and therefore, light illumination causes cantilever deflection [24]. In tapping mode AFM, DC deflection of a cantilever does not affect AFM imaging, so that the photothermal bending of a cantilever should not be a problem as far as its bending is not too large and the light power is stable. In addition, by switching off the illumination light during the trace scan, and by switching it on during the retrace scan [33], we can take optical images without disturbing AFM imaging. However, heating a cantilever causes a problem of increasing the temperature of a solution around the cantilever. Therefore, we have to avoid using UV and near UV lights. 


\section{Maximum imaging rate}

While feedback control to maintain the constant tip-sample interaction force is indispensable for high-speed bioAFM, it limits the imaging rate considerably. The reachable feedback frequency (feedback bandwidth) is determined by the sum of time delays that occur with various devices contained in the feedback loop, such as the cantilever, amplitude detector, feedback controller, and $z$-scanner. The maximum image acquisition rate $V_{\text {image }}$ is estimated from the feedback bandwidth $f_{\mathrm{B}}$, scan range $\left(W_{s}\right)$, and the number of scan lines $\left(N_{\mathrm{s}}\right)$. $V_{\text {image }}$ is approximately given by

$V_{\text {image }}=\frac{w f_{\mathrm{B}}}{2 W_{\mathrm{S}} N_{\mathrm{S}}}=\frac{\alpha w}{16 W_{\mathrm{S}} N_{\mathrm{S}} \sum \tau_{n}}$

where $w$ is the apparent width of features on the surface, $\Sigma \tau_{n}$ is the sum of time delays, $\alpha(\sim 1.4)$ is a factor by which the differential operation contained in feedback control compensates for a phase delay in the feedback loop [20]. $w$ is approximately given by the convolution of the tip radius $r_{\mathrm{t}}$ and the sample radius $r_{\mathrm{s}}$ (i.e., $w=\sqrt{\mathrm{r}_{t} r_{\mathrm{s}}}$ ). Here, the feedback bandwidth is defined as a feedback frequency that results in a phase delay of $45^{\circ}$ in tracing the sample topography. This phase delay produces a feedback error, and therefore, the tip-sample interaction force is not perfectly maintained at a constant level. When the sample is delicate enough to be damaged or disturbed by the extra force caused by the feedback error, $V_{\text {image }}$ should be smaller than that given by Eq. 1. By introducing various devices mentioned below, the feedback bandwidth now stands at $\sim 130 \mathrm{kHz}$, which approximately corresponds to the maximum imaging rate of $\sim 30$ frames/s for a scan range of $\sim 240 \mathrm{~nm}$ and 100 scan lines.

\section{Instrumentation of high-speed AFM}

The basic structure of high-speed AFM is similar to that of conventional AFMs. However, various devices have been optimized for high-speed scanning. The optimized devices are given below.

\section{Cantilevers}

Most advanced cantilevers for high-speed AFM are manufactured by Olympus using silicon nitride [47]. They are $\sim 6 \mu \mathrm{m}$ long, $\sim 2 \mu \mathrm{m}$ wide, and $\sim 90 \mathrm{~nm}$ thick, and are coated with gold of thickness $\sim 20 \mathrm{~nm}$, which results in a resonant frequency $f_{\mathrm{c}}$ of $\sim 3.5 \mathrm{MHz}$ in air and $\sim 1.2 \mathrm{MHz}$ in water, spring constant $k_{\mathrm{c}} \sim 0.2 \mathrm{~N} / \mathrm{m}$, and quality factor $Q_{\mathrm{c}} \sim 2.5$ in water. Therefore, their response time $\tau_{\mathrm{c}}\left(=Q_{\mathrm{c}} / \pi f_{\mathrm{c}}\right)$ is $0.66 \mu \mathrm{s}$ in water. The cantilever tip is not sharp enough $(\sim 17 \mathrm{~nm}$ in radius at the apex). We usually attach an additional tip using electron beam deposition in naphthalene gas and sharpen it ( $\sim \mathrm{nm}$ in radius) by plasma etching in argon gas.

Optical beam deflection detector

To focus an incident laser beam onto a small cantilever, a $\times 20$ objective lens with a long working distance of $8 \mathrm{~mm}$ (Nikon, CFI Plan Fluor ELWD $20 \times \mathrm{C}$; NA, 0.45) is used. The beam reflected back from the cantilever is collected with the same lens. The incident and reflected laser beams are separated using a quarter wavelength plate and a polarization splitter [15-17]. The laser driver is equipped with a radio frequency power modulator for reducing the laser beam coherence [48]. The photosensor consists of a four-segmented Si PIN photodiode ( $3 \mathrm{pF}, 40 \mathrm{MHz}$ ) and a custom-made fast amplifier/signal conditioner $(\sim 20 \mathrm{MHz})$.

\section{Fast amplitude detector}

Conventional rms-to-dc converters use a rectifier circuit and a low-pass filter and require at least several oscillation cycles to output an accurate rms value. To detect the cantilever oscillation amplitude at periodicity of a half oscillation cycle, we developed a method to capture the peak and bottom voltages and then produce their difference as the amplitude [17]. The amplitude detection time $\tau_{\mathrm{d}}\left(=1 / 2 f_{\mathrm{c}}\right)$ is $0.42 \mu \mathrm{s}$ for our small cantilevers with a resonant frequency of $\sim 1.2 \mathrm{MHz}$ in water.

\section{High-speed scanner}

We employed flexure stages made of blade springs for the $x$ and $y$ scanners (Fig. 1). The flexure stages are made by monolithic processing to minimize the number of resonant peaks [22]. The $y$-scanner displaces the $x$-scanner, and the $x$-scanner displaces the $z$-scanner on the top of which a sample stage is placed. The maximum displacements of the $x$ and $y$ scanners at $100 \mathrm{~V}$ are 1 and $3 \mu \mathrm{m}$, respectively. The $x$-piezoactuator is held at both its ends with flexures, so that
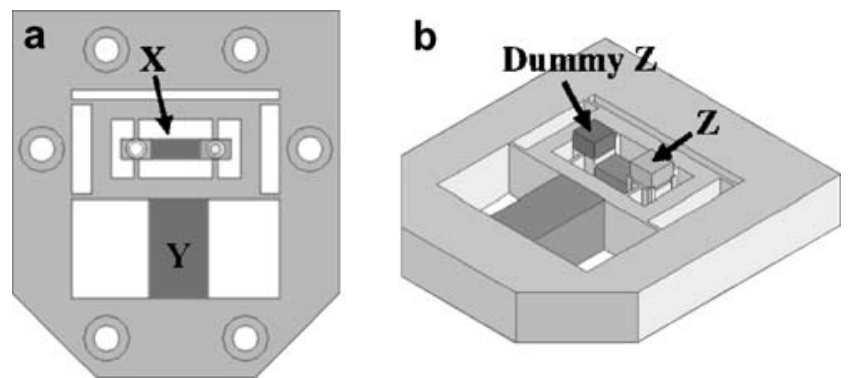

Fig. 1 Scanner structure. a Top view. Note that the $x$-piezoactuator $(\mathrm{X})$ is sandwiched between two sets of flexures. b 3D view. A dummy z-piezoactuator is placed to take a weight balance with the $z$ piezoactuator 
its center of mass is hardly displaced and, consequently, no large mechanical excitation is produced. The $x$-scanner shows resonant peaks at $45,65 \mathrm{kHz}$, and higher frequencies; however, these peaks are not large. The $z$-piezoactuator (maximum displacement, $2 \mu \mathrm{m}$ at $100 \mathrm{~V}$; self-resonant frequency, $360 \mathrm{kHz}$ ) is held only at the four corners of its surface that is perpendicular to the displacement direction. The $z$-piezoactuator can be displaced almost freely in both the counter directions, and consequently, impulsive forces are barely exerted on the holder; thus, only a very low mechanical excitation is produced to the scanner mechanics. This holding method has an additional advantage in that the resonant frequency is not lowered by holding, although the maximum displacement decreases by half. The $x$-scanner is actively damped either by the previously developed $Q$ control technique [19] or by feedforward control using inverse compensation $[49,50]$. The $z$-scanner is also actively damped either by the $Q$-control technique or by inverse compensation. A circuit that automatically produces an inverse transfer function for a given transfer function was developed [24, 51]. By this compensation, the $z$-scanner bandwidth $f_{\mathrm{s}}$ is extended to $\sim 500 \mathrm{kHz}$, and the quality factor $Q_{\mathrm{s}}$ is reduced to $\sim 0.5$. Therefore, its response time $\tau_{\mathrm{s}}\left(=Q_{\mathrm{s}} / \pi f_{s}\right)$ is $\sim 0.32 \mu \mathrm{s}$.

\section{Dynamic PID control}

To observe biological processes that contain delicate protein-protein interactions, the tip-sample interaction force has to be minimized. The tapping force exerted from the oscillating cantilever tip to the sample can be reduced by using an amplitude set point $r$ close to 1 . However, under this condition, the oscillating cantilever tip tends to detach completely from the sample surface at steep down-hill regions of the sample. Once detached, like "parachuting," it takes time for the tip to touch the surface again. Parachuting results in loss of sample topography information and in a low feedback bandwidth. During parachuting, the error signal (difference between the cantilever's peak-to-peak oscillation amplitude $A_{\mathrm{pp}}$ and the peak-to-peak set point amplitude $A_{\mathrm{s}}$ ) is saturated at $\left(2 A_{0}-A_{\mathrm{s}}\right)=2 A_{0}(1-r)$, where $2 A_{0}$ is the free oscillation peak-to-peak amplitude. A shallower set point results in a smaller saturated error signal and hence prolongs the parachuting time $\tau_{\mathrm{p}}$. The feedback gain cannot be increased to shorten $\tau_{\mathrm{p}}$, as a larger gain induces overshoot at up-hill regions of the sample, resulting in the instability of the feedback operation. To solve this problem, we devised a new PID controller named a "dynamic PID controller" that can automatically change the feedback gain depending on the oscillation amplitude [20]. In other words, the feedback gain is increased when $A_{\mathrm{pp}}$ exceeds a threshold level that is set between $A_{\mathrm{s}}$ and $2 A_{0}$ (or at $A_{\mathrm{s}}$ ). By this technique, the parachuting time $\tau_{\mathrm{p}}$ is significantly reduced, or parachuting is avoided completely, resulting in a significant increase in the feedback bandwidth and a decrease in the tapping force.

\section{Drift compensator}

The drift in the cantilever excitation efficiency poses a problem, especially when $A_{\mathrm{s}}$ is set close to $2 A_{0}$. For example, as the efficiency is lowered, $2 A_{0}$ decreases and concomitantly, $A_{p p}$ decreases. The feedback system interprets this decrease in $A_{p p}$ as the tip interacting with the sample too strongly and, therefore, withdraws the sample stage from the cantilever; this, of course, is an incorrect operation. This withdrawal tends to dissociate the cantilever tip completely from the sample surface, especially when $A_{\mathrm{s}}$ is close to $2 A_{0}$. Thus, the dynamic PID controller does not operate properly if the cantilever excitation efficiency is not stabilized. Drifts in the excitation efficiency can be detected by monitoring drifts in the second harmonic amplitude of cantilever oscillation averaged over a time period longer than the image acquisition time [52]. To maintain the constant difference $\left(2 A_{0}-A_{\mathrm{s}}\right)$, we controlled the output gain of a wave generator connected to the excitation piezoactuator [20]. With this drift compensation, very stable imaging was achieved, even with a small difference $\left(2 A_{0}-A_{\mathrm{s}}\right)=$ $0.4 \mathrm{~nm}$.

\section{Other devices (piezo driver, phase detector)}

The piezodriver for the $z$-scanner is custom-made and characterized with a maximum output voltage of $50 \mathrm{~V}$, rms noise $<2 \mathrm{mV}$, high slew rate of $1,000 \mathrm{~V} / \mu \mathrm{s}$, and bandwidth of $3 \mathrm{MHz}$ for capacitive loads up to $2 \mathrm{nF}$. Our small cantilevers have a $\sim 1,000$ times larger value of $f_{\mathrm{c}} / k_{\mathrm{c}}$, when compared with conventional cantilevers. Therefore, a large phase shift occurs by tip-sample interaction even with a small $Q$, showing the potential for fast phase-contrast imaging. We developed a fast-phase detector [53] based on a design by Stark and Guckenberger [54]. This device can detect phase shifts within a single oscillation cycle and, more importantly, at any timing within a cycle. Because of this feature, we can choose the detection timing at the point where the largest phase shift occurs. In addition, it can be inferred whether the tip-sample interactions conserve or dissipate energy. This is because the phase shift due to an energy-conservative interaction decreases very quickly with time, while the phase shift caused by an energy-dissipative interaction is maintained over the oscillation cycle [53].

Photothermal actuation of cantilevers

The tip-sample distance can be controlled not only by the $z$-scanner but also by directly actuating the cantilever. An 
intensity modulated infrared laser beam was used to photothermally deflect small cantilevers [24]. The slow response of the photothermal expansion effect was eliminated by inverse compensation [24]. This method allowed faster control of the tip-sample distance due to the much higher resonant frequency of the small cantilevers than that of a $z$-piezoactuator.

\section{Data acquisition system}

High-speed data acquisition systems (DAQ) are commercially available. We have been using DA/AD boards (10 MHz, 12 bits) with memories of $\sim 0.5 \mathrm{MB} /$ channel and direct access memory (DMA) controller chips, together with our own making software. DMA allows high-speed data transfer without imposing a load to the computer CPU. We can attain a minimum image acquisition time of $\sim 3 \mathrm{~ms}$ using the DA/AD boards. As DAQ with higher rates (100 MHz, 12-16 bits) are available, faster frame acquisition is possible. However, it takes time to display a bitmap image even when data are transferred directly to a bitmap memory area. For example, it takes about $6-10 \mathrm{~ms}$ to display a $256 \times 256$ pixel image. To avoid a time lag between frames caused by this delay, we have to thin out the frames to be displayed. Large amounts of data stream out of high-speed imaging. The maximum number of acquirable successive frames depends on the memory size equipped with the computer. For a typical memory size, 2 GB, of a personal computer, approximately 20,000 successive images with 12 -bit resolution and $256 \times 256$ pixels can be recorded. For a frame rate of $30 \mathrm{~ms} /$ frame, we can record continuously for $10 \mathrm{~min}$. Quantitative descriptions of AFM image acquisition abilities of a given DAQ are given by Fantner et al. [55, 56].

\section{Comparisons of three high-speed AFM Systems}

Three groups (Hansma, Ando, and Miles) have reported instrumentation of high-speed AFM for biological samples. Each AFM setup has unique features, advantages, and drawbacks over the others. Unfortunately, confusion has been occasionally seen in literatures where evaluation and comparison of different setups are described. We cannot simply discuss the comparative merits of high-speed AFM setups just by the achieved imaging rates or scan velocities in the $x$-direction because these quantities depend on various factors that are not necessarily relevant to the instrument performance.

Miles' group has demonstrated ultrafast imaging $(1,300$ frames/s) of collagen fibers in an ambient environment [28]. This remarkable speed was achieved by AFM operation in constant-height mode (without feedback scan of the $z$-scanner), resonant scan of a tuning fork $x$-scanner (resonant frequency, $30-100 \mathrm{kHz} ;[57,58]$ ), and fast digital handling of cantilever deflection data [28]. The cantilevers used are not described well in their articles. The use of a tuning fork as an $x$-scanner was pioneered by Seo et al. $[59,60]$. In an ambient environment, a strong attractive capillary force is produced at thin water layers confined between the tip and the sample. This force avoids the tip being detached from the sample surface even at an extremely high tip velocity of $>4 \mathrm{~cm} / \mathrm{s}$. In addition, this attractive force prevents the dried collagen sample from being damaged by the rapidly scanning tip. However, their high-speed AFM is limited to samples firmly attached to a substratum in an ambient environment and, therefore, does not seem applicable to the observation of biomolecular processes.

Hansma's group has developed various devices for highspeed AFM; fast scanners [56, 61], data acquisition systems $[55,56]$, an optical deflection detection system [15] for small cantilevers, and two types of small cantilevers [56]. Their designs seem to be made with intention of making them compatible with the Veeco Nanoscope. Their small cantilevers for contact mode imaging have a resonant frequency of $150-350 \mathrm{kHz}$ in air and a spring constant of $1-5 \mathrm{pN} / \mathrm{nm}$, while those for tapping mode imaging have a resonant frequency of up to $2 \mathrm{MHz}$ in air and a spring constant below $2 \mathrm{~N} / \mathrm{m}$. Their scanners are designed with intention of observing relatively wide areas; a scan range of $6-15 \mu \mathrm{m}$ in $x$ - and $y$-directions and $6 \mu \mathrm{m}$ in $z$-direction. The scanners are equipped with strain gauge sensors for linearizing the displacements by a closed loop controller. Their lowest resonant frequency is $10-28 \mathrm{kHz}$. However, it is not clear from their papers how high resonant frequency is attained for the most important $z$-scanner. Judging from the piezoactuators used in the $z$-scanner, it must be around $60 \mathrm{kHz}$ (a few times lower than that of our $z$-scanner). It is also not clear whether an active damping controller is implemented in the $z$-scanner. As the digital feedback system implemented in the Veeco Nanoscope III is used, the developed devices do not seem to be effectively utilized to achieve high imaging rates. Thus, their images of biological samples taken in a sub-second timescale were obtained in the so-called error mode (error signals are used to form images; [56, 62]).

As described in detail in the previous section, Ando's group has been developing various devices with strong intention of realizing video rate imaging of biomolecular processes. We have developed the whole high-speed AFM setups without considering their compatibility to commercial AFMs. We have been concentrating on increasing the feedback bandwidth as high as possible. We also have been making efforts for low tapping forces exerted from an oscillating tip to the sample. Sacrificing the scan range to 
achieve a high feedback bandwidth is inevitable at present. However, this issue may be resolved as discussed latter.

\section{Imaging of biomolecular processes}

High-speed AFM is not completely established yet as a tool for studying biomolecular processes. Therefore, in the present stage, biological processes that have been expected or known to occur have to be demonstrated by high-speed AFM. In addition, techniques for preparing samples and substrates for their attachment have to be developed; these techniques are often different from those for still imaging. Along with the gradual accumulation of successful imaging of known molecular processes, high-speed AFM will be considered a reliable tool, while newly filmed data on unexplored biological processes will be widely accepted. The imaging studies that have been performed by us and others are described below.

\section{Early imaging studies}

Early imaging studies with high-speed AFM were performed mainly for evaluating the capacity of this new microscope and for finding devices for improvement. Soon after the first successful imaging of moving myosin molecules at $80 \mathrm{~ms} /$ frame [17], it was realized that the tip-sample interaction force was too strong to image fragile samples such as actin filaments and microtubules, which were easily disrupted during imaging (Fig. 2). The disruption of fragile samples was not only due to the insufficient feedback speed but also due to the large tapping force exerted from the oscillating tip to the sample. Using a dynamic PID controller, the tapping force was greatly reduced. For example, the unidirectional movement of individual kinesin molecules along microtubules [21] was observed (Fig. 3) without microtubule disruption. However, in the observed kinesin movement, the kinesin molecules were attached to both the mica surface and microtubules. The movement of kinesin that was attached only to microtubules was not observed, implying that the tip removed kinesin that had attached only to microtubules. In the observation of actin filament movement, we

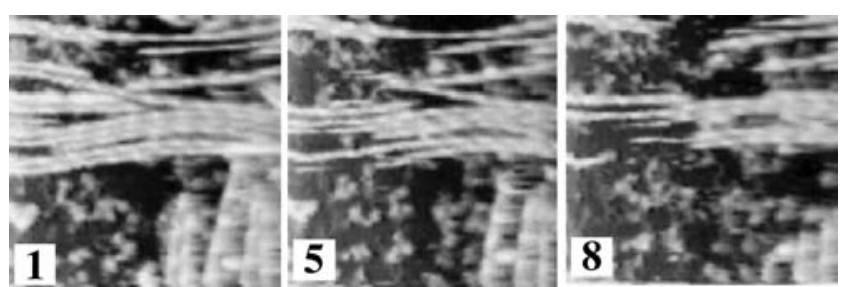

Fig. 2 Actin filaments being disrupted during imaging. The number attached to each image indicates the time (s) elapsed after imaging began. Imaging rate, $80 \mathrm{~ms} /$ frame; scan size, $400 \mathrm{~nm}$

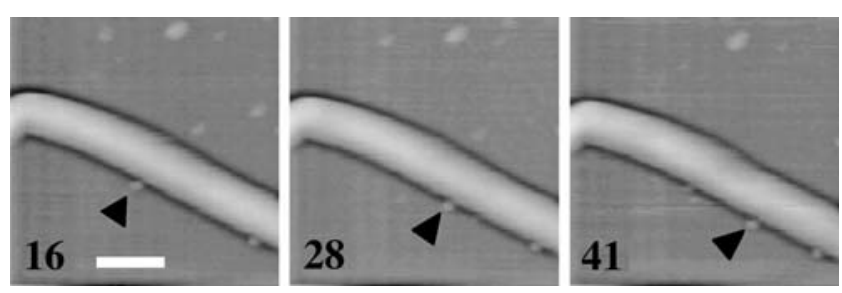

Fig. 3 Movement of kinesin-gelsolin along a microtubule. The number attached to each image indicates the time (s) elapsed after imaging began. The arrowheads point at kinesin-gelsolin. Scale bar, $100 \mathrm{~nm}$; imaging rate, $0.64 \mathrm{~s} /$ frame

experienced a similar problem. When the mica surface was densely coated with myosin $\mathrm{V}$, the gliding of actin filaments was captured on video (Fig. 4; [22, 23]). However, when the myosin $\mathrm{V}$ density was lowered, the gliding movement was hardly observed. These observations indicated that with the assistance of a dynamic PID controller, the tip-sample interaction force was successfully reduced to a level at which protein denaturation was prevented and self-assembled polymer structures were not disrupted. However, the force was not reduced sufficiently to prevent the delicate protein-protein association from breaking.

It is important to observe how protein conformation changes dynamically upon binding to its ligand. Protein molecules always show Brownian motion due to the thermal agitation of surrounding water molecules. Therefore, changes in protein shape caused by this random agitation have to be distinguished from ligand-induced conformational changes. When the ligand associationdissociation kinetics is fast, the ligand-induced changes occur repeatedly. A certain type of structural change that is repeatedly observed can be judged as one induced by the reaction. On the other hand, when the kinetics is slow, this distinction is difficult. For example, due to the low ATPase activity of myosin $\mathrm{V}$ alone $(\sim 0.05 / \mathrm{s})$, it was difficult to judge whether the structural changes observed just once or twice were, in fact, those induced by the binding of ATP (or its hydrolysis; Fig. 5).

To solve this problem, we combined a UV-flash photolysis of caged compounds with high-speed AFM. However, we first encountered a following technical
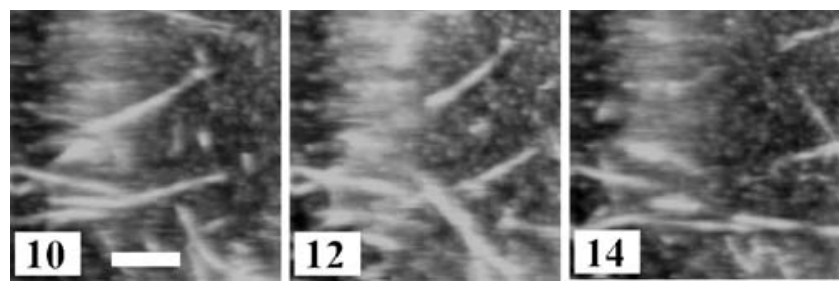

Fig. 4 Actin filaments gliding over a surface that are densely coated with myosin $\mathrm{V}$. The number attached to each image indicates the time (s) elapsed after imaging began. Scale bar, $200 \mathrm{~nm}$; imaging rate, $1 \mathrm{~s} /$ frame 


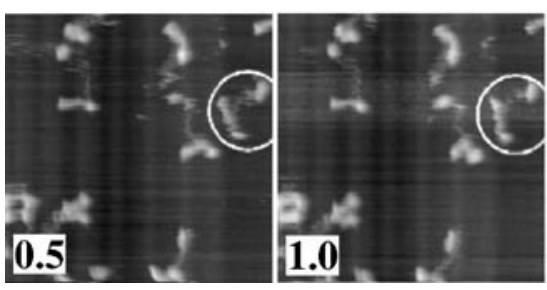

Fig. 5 Myosin V in ATP. The number attached to each image indicates the time (s) elapsed after imaging began. The head portion of myosin $\mathrm{V}$ molecule, which is circled, seems to change its conforma-

problem. An intense flash illumination by a $355-\mathrm{nm}$ laser caused large bending of the cantilever due to the photothermal expansion effect. This caused the tip to hit the substratum with a strong force, leading to tip damage. To solve this problem, attenuated high-frequency laser pulses $(\sim 50 \mathrm{kHz})$ were applied, while the $y$-scanner was being scanned towards the starting point after the completion of one frame acquisition. In addition, during this period, the sample stage was withdrawn from the cantilever tip. This combined method allowed us to observe the rotational movement of the myosin $\mathrm{V}$ head around the head-neck junction that occurred immediately after UV application (Fig. 6). We also applied this method to observe the height changes in GroEL upon binding with ATP and binding with GroES.

Single-headed dynein C from chlamydomonous flagella has a ring-shaped head from which a stem and a stalk protrude. The stalk interacts with microtubules at its globular distal end. Previously, electron micrographs of dynein $\mathrm{C}$ revealed that the angle between the stalk and the stem differed between the no-nucleotide state and the ADPVi bound state [63]. We confirmed this result using AFM in aqueous solutions. As dynein $\mathrm{C}$ has a relatively high ATPase rate even in the absence of microtubules, it must be easy to distinguish the ATPase-driven conformational change from its Brownian motion. In fact, high-speed AFM imaging of dynein $\mathrm{C}$ alone in the presence of ATP revealed that the stem moved back and forth between two positions while the stalk and head were stationary [22, 23]. The two positions approximately corresponded to the nucleotide-free and ADP-Vi bound states. Besides, the frequency of the stem movement was similar to the ATPase rate. Therefore,

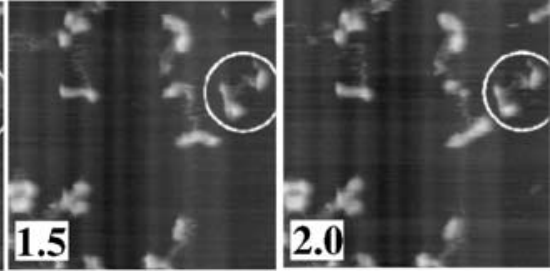

tion from an extended form to a folded form. ATP concentration is $2 \mu \mathrm{M}$. Imaging rate, $0.5 \mathrm{~s} /$ frame; scan range, $300 \mathrm{~nm}$

it seems certain that the observed periodic stem movement is actually driven by the ATPase reaction.

Recent imaging studies

We learned from early imaging studies that reduction in the tapping force was essential to the establishment of highspeed AFM truly useful in biological research. To make dynamic PID control more effective, we developed a lownoise sensor amplifier. Due to the low noise, we could use an amplitude set point of $r>0.9$, which made it possible to observe actin filament gliding on a mica surface sparsely coated with myosin V (Fig. 7). The bound heads of myosin $\mathrm{V}$ were oriented along one direction [22, 23], like the wellknown arrowhead structure in muscles. From this structure, the sliding actin filament polarity was identified, and thus, it was shown that this filament moved toward the minus end (i.e., natural direction). However, we could not detect large conformational changes in the bound heads while the actin filament was moving unidirectionally.

In spite of this successful observation, we were unable to observe the processive movement by single myosin $\mathrm{V}$ molecules along actin filaments. In low ionic strength solutions, myosin $\mathrm{V}$ tended to attach to the mica surface. In high ionic strength solutions, it was nearly free from the surface, but the affinity of myosin $\mathrm{V}$ for actin was lowered. To circumvent this problem, we reduced the cantilever-free oscillation amplitude from the usual $\sim 5$ to $\sim 1 \mathrm{~nm}$, thereby, sacrificing the feedback bandwidth. With such a small amplitude and in a high ionic strength solution containing a low concentration of ATP, the processive movement was finally captured on video at $0.1 \mathrm{~s} /$ frame. The two heads of a
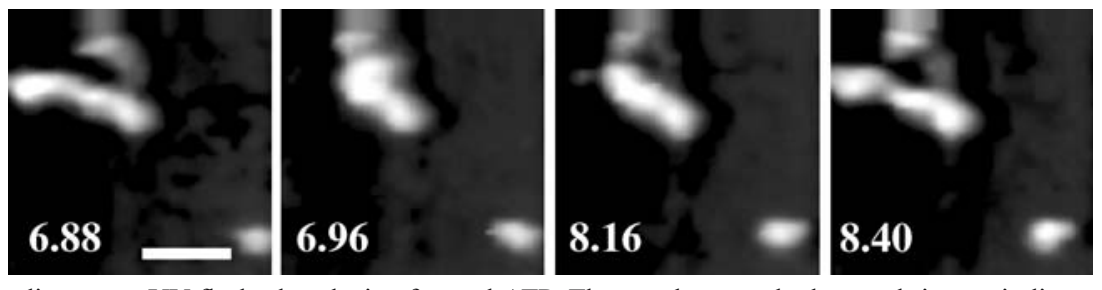

Fig. 6 Myosin V head bending upon UV-flash photolysis of caged ATP. The number attached to each image indicates the time (s) elapsed after imaging began. UV flash was applied between the first and second images. Scale bar, $30 \mathrm{~nm}$; imaging rate, $80 \mathrm{~ms} /$ frame 


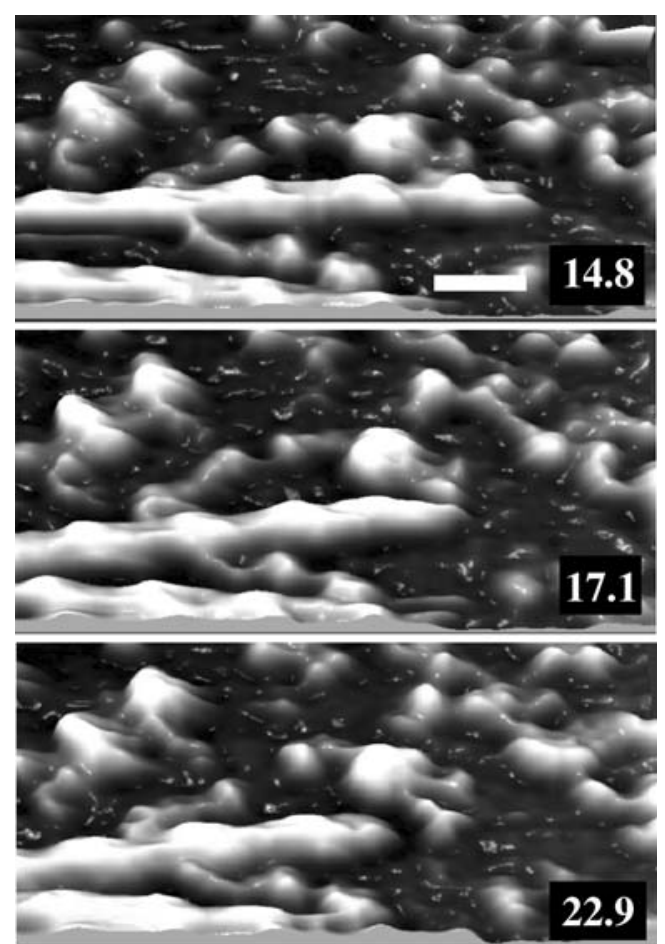

Fig. 7 Three-dimensional images of actin filament sliding movement captured by high-speed AFM. The number attached to each image indicates the time (s) elapsed after imaging began. Scale bar $30 \mathrm{~nm}$, imaging rate, $180 \mathrm{~ms} /$ frame

myosin $\mathrm{V}$ molecule were changing the leading and trailing positions alternately with a walking stride of $\sim 72 \mathrm{~nm}$, i.e., in a hand-over-hand manner as demonstrated by singlemolecule fluorescence microscopy [64-67]. High-speed AFM revealed the movement in greater detail than that shown by fluorescence microscopy. The lead lever-arm bent just before the rear head detached from the actin filament, as previously shown by electron microscopy [68]. The detached rear head rotated around the junction between the two lever arms and then attached to a frontward actin. Just after the attachment, the new lead head moved farther by $\sim 5 \mathrm{~nm}$ along the actin filament. In the absence of nucleotide or in the presence of ADP, only one head of a myosin V molecule attached to an actin filament. From the orientation of the bound head, it was clear that the bound head was in the trailing position. On the other hand, in the presence of a medium concentration of AMP-PNP, both heads were associated with an actin filament for a long time. This indicates that on binding to AMP-PNP, the lead head conformation adapts itself so that it is able to bind to actin by rotating around the head-neck junction. Therefore, with myosin V that is bound to an actin filament at both the heads in an ATP-containing solution, the trailing head must contain ADP (or no nucleotide in a low ATP concentration), and the lead head must contain ATP or ADP-Pi. After the phosphate is released, the lead head rotates back to the previous orientation, an orientation unfavorable for actin binding. However, the lead head cannot dissociate from actin because the ADP-bound state has a high affinity for actin. This energetically unfavorable conformation bends the lead neck forward, thereby pulling the trailing head, so that it detaches from actin and then being conveyed frontward. Using these mechanical processes along the chemical reaction pathway, the two heads alternate their positions to walk along an actin filament.

Another example of biomolecular processes successfully captured is GroEL-GroES association-dissociation cycles controlled by the ATPase reaction. GroEL consists of 14 identical ATPase subunits that form two heptameric rings stacked back to back $[69,70]$. From a series of biochemical studies [71, 72], it has been shown that there is positive cooperativity for binding and hydrolysis of ATP in the same ring and negative cooperativity between the two rings. Due to this negative cooperativity, it has been considered that GroEL binds with GroES at one ring while releasing GroES from the other [73-75]. This alternate on-off switching seems to exclude the concomitant binding of GroES to both rings of GroEL. However, this issue has remained controversial [76-80], because some electron micrographs have shown a complex of GroES-GroELGroES with a football shape. To place GroEL on a substratum in a side-on orientation so that both the rings are accessible to GroES, we prepared GroEL that were biotinylated at the equatorial domains [81]. The GroEL was attached to streptavidin 2D crystal sheets prepared on planar lipid bilayers containing a biotin lipid. We could capture the alternate on-off events. Surprisingly, before the alternate switching took place, a football structure appeared with a high probability. Thus, the high-speed AFM observation clearly solved the controversial issue.

\section{Imaging with commercial high-speed AFM}

Infinitesima (Oxford, U.K.) has recently produced Video$\mathrm{AFM}^{\mathrm{TM}}$ equipped with a fast $x$-scanner made of a quartz tuning fork, based on the studies by Miles' group [57, 58]. A fast feedback system is not implemented to this commercial high-speed AFM. Although its marketing is restricted only in Japan at present, Olympus (Tokyo, Japan) has produced Nano Live Vision ${ }^{\mathrm{TM}}$ (Olympus, Tokyo, Japan), which is a modified version of the microscope developed by Ando's group [17]. Users of the latter product recently filmed dynamic processes of GroEL-GroES interaction that were regulated by the ATPase reaction of GroEL immobilized onto mica surface in an end-up orientation [82]. Moreover, they observed formation and dissociation of a streptavidin-biotinylated DNA complex [83] and one-dimensional diffusion of a restriction enzyme along a DNA strand which was followed by the cleavage reaction [84]. A most recent study successfully captured 
translocation and looping of DNA by a type III restriction enzyme, EcoP15I [85].

\section{Substratum}

The choice of the substratum on which a sample is placed is very important to the AFM observation of biomolecular processes. The preparation of substrata with very small roughness have been explored extensively for still-image observations [86]. However, these preparations have mainly been devised to attach samples firmly onto them. Therefore, they are often useless for visualizing biomolecular processes. The visualization of biomolecular processes requires the substratum to have various properties; for example, (a) appropriate binding affinity for the sample to ensure that its physiological function is retained, (b) selective attachment of a specific component in a multicomponent sample, and (c) attachment of molecules in the desired orientation. Mica (natural muscovite or synthetic fluorophlogopite) has been frequently used as a source substratum due to its surface flatness at the atomic level over a large area. It has net negative charges and therefore is quite hydrophilic. A bare mica surface adsorbs various proteins by electrostatic interaction. Except for some cases (such as GroEL attachment in an end-up orientation), its binding orientation is not unique, and selective attachment of a specific species is not expected.

If possible, it is best to have, in the first step, a surface on which the sample never attaches. Then, this surface is modified so that only a specific species of molecules can attach to it through a linker in a desired orientation. A membrane surface with zwitterionic polar head groups such as phosphatidyl choline (PC) and phosphatidyl ethanolamine (PE) is known to resist protein adsorption [87, 88]. Streptavidin is also useful for specific attachment of biotinylated protein because it also resists nonspecific protein adsorption. Mica-surface-supported planar lipid bilayers [89] can be easily formed from liposomes as their strong hydrophilic interaction disrupts the liposomes [90]. Various lipids with functional groups that are attached to polar groups (e.g., biotin attached to PE, Ni-NTA attached to GS) are available. They enable the specific attachment of proteins labeled with biotin or his-tags onto planar lipid bilayers. DOPC lipid is useful for the preparation of streptavidin 2D crystals when it is used together with biotinylated lipids [91, 92]. It contains an unsaturated hydrocarbon on each of the two alkyl chains, which causes the bending of the chains and, therefore, weakens the interaction between neighboring DOPCs. This weak interaction lowers the phase-transition temperature of DOPC lipid down to approximately $-20^{\circ} \mathrm{C}$, thereby affording large fluidity to the planar bilayer at room temperature and facilitating 2D-crystal formation of streptavidin (Fig. 8a). The densely packed streptavidin does not diffuse easily. If less diffusibility is necessary, the packed streptavidin can be cross-linked using glutaraldehyde, which does not influence its ability to bind to biotin. We used this surface for the selective attachment of biotinylated GroEL in a side-on orientation. DPPC contains no unsaturated hydrocarbons in the alkyl chains; therefore, its phase-transition temperature is high $\left(\sim 41^{\circ} \mathrm{C}\right)$, and it is appropriate for preparing planar bilayers with low fluidity. For example, when planar bilayers are formed with DPPC at a high temperature $\left(\sim 60^{\circ} \mathrm{C}\right)$ together with a certain fraction of DPPE-biotin, streptavidin, which is sparsely attached to the surface, hardly diffuses at room temperature (Fig. 8b). When DOPE-biotin is used together with DPPC, the sparsely attached streptavidin diffuses at a moderate rate (Fig. 8c).

a

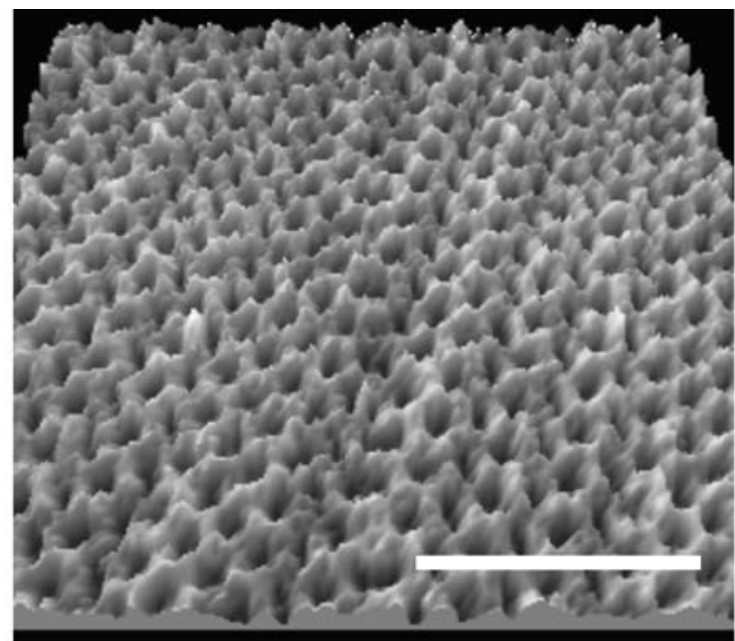

b
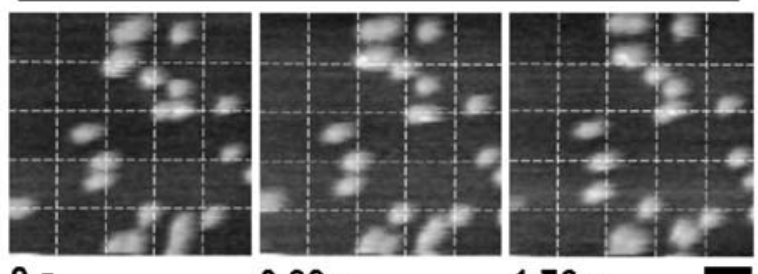

$0 \mathrm{~s}$

$0.88 \mathrm{~s}$

$1.76 \mathrm{~s}$

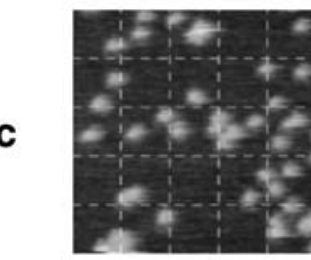

$0 \mathrm{~s}$

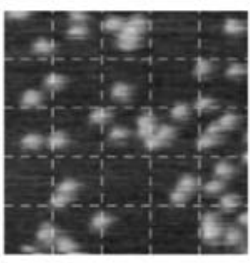

$0.18 \mathrm{~s}$

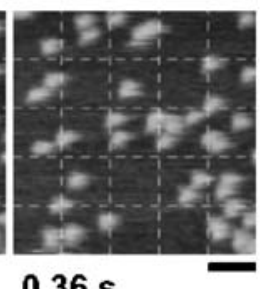

$0.36 \mathrm{~s}$

Fig. 8 Streptavidin on mica-supported planar lipid bilayers. (a) Streptavidin 2D crystals formed on bilayers of DOPC+DOPE-biotin. Scale bar, $30 \mathrm{~nm}$. (b) Streptavidin on bilayers of DPPC+DPPE-biotin. Scale bar, $40 \mathrm{~nm}$ : imaging rate, $0.18 \mathrm{~s} /$ frame. (c) Streptavidin on bilayers of DPPC+DOPE-biotin. Scale bar, $40 \mathrm{~nm}$; imaging rate, $0.18 \mathrm{~s} /$ frame 


\section{Future prospects}

Speed and interaction force

As demonstrated in the successful imaging mentioned above, high-speed AFM is becoming a useful tool in biological sciences, particularly in the study of the molecular processes of proteins. To expand the scope of biological samples that can be studied by high-speed AFM, the imaging rate must be enhanced further and the tipsample interaction force must be significantly reduced. At present, the cantilever is the main factor that limits both these capabilities. Most advanced small cantilevers developed by Olympus seem to have almost achieved the ultimate goal of balancing a high resonant frequency and a small spring constant. The question therefore is how this seemingly impenetrable level can be surpassed.

Tip-sample interaction has been detected by measuring the changes in the amplitude, phase, or resonant frequency of an oscillating cantilever. With the small quality factor of the cantilever that is necessary for obtaining a fast response, these three quantities are not sensitive to the impulsive interaction. In tapping mode AFM, where the excitation frequency is fixed, a change in the resonant frequency is detected as the phase shift relative to the excitation signal. For a cantilever with a low quality factor, the phase does not change sharply as a function of the resonance frequency, resulting in low sensitivity of the phase to the interaction. The influence of an impulse ( p peak force $\times$ interaction time) on the cantilever is distributed over harmonic frequencies (integral multiples of the fundamental frequency). The cantilever oscillation gain is lower at higher harmonic frequencies, which gives rise to the low sensitivity of the amplitude. As the impulsive force is exerted transiently in a short time, its peak force is relatively large. This means that the peak force must be a highly sensitive quantity. Although the force $F(t)$ cannot be detected directly and precisely, a rough estimate of $F(t)$ can be obtained from the cantilever's oscillation waves $z(t)$ by putting $z(t)$ into the cantilever motion equation and then subtracting the excitation signal (i.e., inverse determination problem; [93]). Here, we do not need to resort the differential of $z(\mathrm{t})$, a process that yields noisy signals. An operation where the phase of each Fourier decomposed harmonic signal is shifted by $\pi / 2$ and then multiplied by an appropriate gain is identical to differentiation. To make this method effective, the cantilever's oscillation signal must be detected with a wide bandwidth (at least up to $4 \times f_{\mathrm{c}}$ ), and fast analog or digital calculation systems are necessary for converting $z(t)$ to $F(t)$. In addition, a fast peak-hold system is necessary to capture the peak force. Alternatively, the peak force can be calculated using only Fourier coefficients, without obtaining the force signal as a function of time. This sensitive method for detecting the tip-sample interaction should lower the interaction force.

The ultimate way to minimize the interaction force is noncontact imaging. Nc-AFM has been realized only in vacuum by utilizing a significantly large quality factor of the cantilever in vacuum. If high-speed nc-AFM is realized in liquid conditions, we can use stiffer cantilevers with higher resonant frequencies, which can promise higher imaging rates. In addition, high-speed nc-AFM will make it possible to image dynamic molecular processes that occur on living cell membranes, a process that is, at present, absolutely impossible, as living cell membranes are extremely soft. As many important proteins exist on membranes, noncontact imaging capability greatly expands the usefulness of highspeed AFM in life sciences. Extracellular stimuli (such as drugs, endogenous ligands, or electric one) are first received by membranes proteins (receptors or ionic channels) and then transmitted to the interiors to produce various cellular actions. Various diseases are linked with membrane proteins, sugar chains in membrane glycoproteins, and several intracellular molecules involved in signal transductions. Therefore, high-speed nc-AFM will also make a great contribution to disease detection, diagnosis, and drug assessment and discovery.

An ultrasonic technique may have the potential of realizing noncontact imaging; here, the cantilever tip and the sample are ultrasonically excited at different frequencies of $f_{1}$ and $f_{2}\left(f_{1}, f_{2}>>f_{c}\right)$ with $\left|f_{1}-f_{2}\right| \sim f_{c}$. Even when the tip is not in contact with but close to the sample surface, the two ultrasound waves must interfere with each other to produce acoustic waves with a frequency of $\left|f_{1}-f_{2}\right|$ that excites the cantilever. A similar configuration has been used for scanning near-field ultrasound holography (SNFUH), which has been developed for high-resolution subsurface imaging [94]. Ion-conductance scanning probe microscopy (ICSPM) already satisfies the noncontact condition [95]. To materialize high-speed ICSPM, we must find a method to increase the bandwidth of ion-conductance detection, as ionic currents through a small pore of the capillary electrode are very low.

\section{High-speed AFM for intracellular imaging}

SNFUH has already successfully been used in intracellular imaging [94]. In its application, a high-frequency acoustic wave is launched from under the sample stage, and this wave then propagates through the sample. Materials with different elastic moduli embedded in the sample modulate the phase and amplitude of the propagating acoustic waves. These modulations are reflected on the acoustic interference that occurs at the cantilever tip and, consequently, are reflected on the cantilever oscillation around its resonant frequency. It does not seem difficult to combine SNFUH 
with the high-speed scanning techniques developed so far. This combination will enable the filming of dynamic processes of intracellular organelles. Although it is presently unclear whether SNFUH has resolution in the $z$-direction, the images obtained with ultrasonic waves launched at different angles should contain information along the $z$-axis. Therefore, there may be a way to reconstitute a $3 \mathrm{D}$ image from multiple images obtained at different launching angles. High-resolution 3D imaging of intracellular structures in real time will revolutionize cell biology and physiology. Various intracellular processes in living cells and organisms that take place spontaneously or responding to extracellular stimuli may be able to be examined by high-speed SNFUH combined with optical microscopy.

\section{High-speed recognition AFM}

Hinterdorfer and his colleagues recently developed a recognition imaging technique $[31,32]$. In their technique, a cantilever tip that is functionalized with a probe molecule through a flexible short-linker is oscillated at a frequency below resonance. Its oscillation is split into approaching and withdrawing parts. The oscillation signals in the approaching part reflect the repulsive tip-sample interaction, providing topography images. The signals in the withdrawing part reflect the association events between the probe molecule and its counterpart, providing recognition images. The effective concentration of a probe molecule attached to a cantilever tip depends on the tether length. With a tether length of $2 \mathrm{~nm}$, the concentration is $\sim 50 \mathrm{mM}$, which is high enough for the association reaction to take place in $20 \mu$ s for a system with a typical association rate constant of $1 \times 10^{6} \mathrm{M}^{-1} \mathrm{~s}^{-1}$. This suggests that recognition imaging at a moderate rate $(\sim 0.4 \mathrm{~s} /$ frame $)$ is possible so long as the cantilever oscillation amplitude is small. Another type of recognition imaging may be derived from phase-contrast imaging [53] using a cantilever tip functionalized with a specific probe molecule. Its phasecontrast image will reveal the arrangement of the specific molecules in the sample.

\section{High-resolution and high-speed AFM}

Atomic resolution had been attained with AFM only in ultrahigh vacuum environments. However, recent studies with frequency-modulation AFM (FM-AFM) have achieved atomic resolution in a liquid environment by minimizing the sensor noise and using a small amplitude of cantilever oscillation $[48,96]$. In addition, the highresolution imaging capability of FM-AFM was also demonstrated by successful imaging of individual water layers weakly interacting with lipid membrane surfaces
[97]. In these studies, conventional cantilevers with a relatively small quality factor $(\sim 6)$ were used. As a much larger shift in the resonant frequency is expected to occur with small cantilevers, it seems possible to achieve subnanometer resolution as well with high-speed tapping mode AFM equipped with a fast frequency-shift (phase-shift) detector [53] and a sharp cantilever tip. Our recent preliminary experiments filmed atomic resolution images of a mica surface in water at a frame rate of 37 frames/s, although further noise reduction remained to be accomplished. AFM with both high-resolution and high-speed capabilities has enormous potential; imaging of secondary structures on protein surfaces and their dynamic changes, mapping of rapid changes in the water layer structure at protein surfaces, imaging of dynamic lipid-protein interaction on membranes, and detection of small-ligand interaction with its active site on a protein surface. It is impossible to study these phenomena by using "conventional" highspeed AFM.

Direct seeing at in-liquid nanometer-scale worlds in real time will markedly change our style of examining biological problems. As discussed above, the usefulness of high-speed imaging capability of AFM will effectively expand by other techniques being combined. In the initial stage, high-speed AFM will be actively used to observe a wide range of dynamic processes with isolated proteins, protein assemblies, and protein-DNA complexes. In the next stage, more complex systems including living cells and organisms will become targets of high-speed AFM combined with other techniques. In vivo and in vitro visualization of various processes at the molecular level will become possible; responses of membrane receptors to stimuli, nuclear envelope formation and disassembly, chromosome replication and segregation processes, phagocytosis, proteins synthesis in the endoplasmic reticulum, targeting processes of synthesized proteins through the Golgi apparatus, and etc. Thus, high-speed AFM-based visualization techniques have a great potential of innovating not only in biochemistry and biophysics but also in cell biology, physiology, and pharmaceutical and medical sciences. To open up such unprecedented fields, steady efforts have to be carried out towards expanding the capability of highspeed AFM and the related techniques.

\section{References}

1. Sako Y, Yanagida T (2003) Review: single-molecule visualization in cell biology. Nature Rev Mol Cell Biol 4:SS1-SS5

2. Wallace MI, Molloy JE, Trentham DR (2003) Minireview: combined single-molecule force and fluorescence measurements for biology. J Biol 2(1):4, (5pp) 
3. Cornish PV, Ha T (2007) A survey of single-molecule techniques in chemical biology. ACS Chem Biol 2:53-61

4. Drake B, Prater CB, Weisenhorn AL, Gould SAC, Albrecht TR, Quate CF, Cannell DS, Hansma HG, Hansma PK (1989) Imaging crystals, polymers, and processes in water with the atomic force microscope. Science 243:1586-1589

5. Zhong Q, Inniss D, Kjoller K, Elings VB (1993) Fractured polymer/silica fiber surfaces studied by tapping mode atomic force microscopy. Sur Sci 290:L688-L692

6. Kasas S, Thomson NH, Smith BL, Hansma HG, Zhu X, Guthold M, Bustamante C, Kool ET, Kashlev M, Hansma PK (1997) Escherichia coli RNA polymerase activity observed using atomic force microscopy. Biochemistry 36:461-468

7. Guthold M, Zhu X, Rivetti C, Yang G, Thomson NH, Kasas S, Hansma HG, Smith B, Hansma PK, Bustamante C (1999) Direct observation of one-dimensional diffusion and transcription by Escherichia coli RNA polymerase. Biophys. J 77:2284-2294

8. Barrett RC, Quate CF (1991) High-speed, large-scale imaging with the atomic force microscope. J Vac Sci Technol B 9:302-306

9. Sulchek T, Minne SC, Adams JD, Fletcher DA, Atalar A, Quate CF (1999) Dual integrated actuators for extended range high speed atomic force microscopy. Appl Phys Lett 75:1637-1639

10. Rogers B, Manning L, Sulchek T, Adams JD (2004) Improving tapping mode atomic force microscopy with piezoelectric cantilevers. Ultramicroscopy 100:267-276

11. Minne SC, Yaralioglu G, Manalis SR, Adams JD, Zesch J, Atalar A, Quate CF (1998) Automated parallel high speed atomic force microscopy. Appl Phys Lett 72:2340-2342

12. Minne SC, Adames JD, Yaralioglu G, Manalis SR, Atalar A, Quate CF (1998) Centimeter scale atomic force microscope imaging and lithography. Appl Phys Lett 73:1742-1744

13. Sulchek T, Grow RJ, Yaralioglu GG, Minne SC, Quate CF, Manalis SR, Kiraz A, Aydine A, Atalar A (2001) Parallel atomic force microscopy with optical interferometric detection. Appl Phys Lett 78:1787-1789

14. Rogers B, Sulchek T, Murray K, York D, Jones M, Manning L, Malekos S, Beneschott B, Adams JD, Cavazos H, Minne SC (2003) High speed tapping mode atomic force microscopy in liquid using an insulated piezoelectric cantilever. Rev Sci Instrum 74:4683-4686

15. Viani MB, Schäffer TE, Paloczi GT, Pietrasanta LI, Smith BL, Thompson JB, Richter M, Rief M, Gaub HE, Plaxco KW, Cleland AN, Hansma HG, Hansma PK (1999) Fast imaging and fast force spectroscopy of single biopolymers with a new atomic force microscope designed for small cantilevers. Rev Sci Instrum 70:4300-4303

16. Schäffer TE, Cleveland JP, Ohnesorge F, Walters DA, Hansma PK (1996) Studies of vibrating atomic force microscope cantilevers in liquid. J Appl Phys 80:3622-3627

17. Ando T, Kodera N, Takai E, Maruyama D, Saito K, Toda A (2001) A high-speed Atomic force microscope for studying biological macromolecules. Proc Natl Acad Sci USA 98:12468-12472

18. Ando T, Kodera N, Maruyama D, Takai E, Saito K, Toda A (2002) A high-speed atomic force microscope for studying biological macromolecules in action. Jpn J Appl Phys 41:48514856

19. Kodera N, Yamashita H, Ando T (2005) Active damping of the scanner for high-speed atomic force microscopy. Rev Sci Instrum 76:053708, (5pp)

20. Kodera N, Sakashita M, Ando T (2006) Dynamic proportionalintegral-differential controller for high-speed atomic force microscopy. Rev Sci Instrum 77:083704, (7pp)

21. Ando T, Kodera N, Naito Y, Kinoshita T, Furuta K, Toyoshima YY (2003) A high-speed atomic force microscope for studying biological macromolecules in action. Chem Phys Chem 4:11961202
22. Ando T, Uchihashi T, Kodera N, Miyagi A, Nakakita R, Yamashita H, Matada K (2005) High-speed atomic force microscopy for capturing dynamic behavior of protein molecules at work. e-J. Surf Sci Nanotech 3:384-392

23. Ando T, Uchihashi T, Kodera N, Miyagi A, Nakakita R, Yamashita H, Sakashita M (2006) High-speed atomic force microscopy for studying the dynamic behavior of protein molecules at work. Jpn J Appl Phys 45:1897-1903

24. Yamashita H, Kodera N, Miyagi A, Uchihashi T, Yamamoto D, Ando T (2007) Tip-sample distance control using photo-thermal actuation of a small cantilever for high-speed atomic force microscopy. Rev Sci Instrum 78:083702, (5pp)

25. Hell SW, Dyba M, Jakobs S (2004) Concepts for nanoscale resolution in fluorescence microscopy. Curr Opin Neurobiol 14:599-609

26. Donnert G, Keller J, Medda R, Andrei MA, Rizzoli SO, Lührmann R, Jahn R, Eggeling C, Hell SW (2006) Macromolecular-scale resolution in biological fluorescence microscopy. Proc Natl Acad Sci USA 103:11440-11445

27. Funatsu T, Harada Y, Tokunaga M, Saito K, Yanagida T (1995) Imaging of single fluorescent molecules and individual ATP turnovers by single myosin molecules in aqueous solution. Nature 374:555-559

28. Picco LM, Bozec L, Ulcinas A, Engledew DJ, Antognozzi M, Horton MA, Miles MJ (2007) Breaking the speed limit with atomic force microscopy. Nanotechnology 18:044030, (4pp)

29. Giessibl FJ (1995) Atomic resolution of the silicon $(111)-(7 \times 7)$ surface by atomic force microscopy. Science 267:68-71

30. Ishijima A, Kojima H, Funatsu T, Tokunaga M, Higuchi H, Tanaka H, Yanagida T (1998) Simultaneous observation of individual ATPase and mechanical events by a single myosin molecule during interaction with actin. Cell 92:161-171

31. Stroh C, Wang H, Bash R, Ashcroft B, Nelson J, Gruber H, Lohr D, Lindsay SM, Hinterdorfer P (2004) Single-molecule recognition imaging microscopy. Proc Natl Acad Sci USA 101:1250312507

32. Hinterdorfer P, Dufrêne YF (2006) Detection and localization of single molecular recognition events using atomic force microscopy. Nature Methods 3:347-355

33. Kassies R, Van Der Werf KO, Lenferink A, Hunter CN, Olsen JD, Subramaniam V, Otto C (2005) Combined AFM and confocal fluorescence microscope for applications in bio-nanotechnology. $\mathrm{J}$ Microsc 217:109-116

34. Meller K, Theiss C (2006) Atomic force microscopy and confocal laser scanning microscopy on the cytoskeleton of permeabilised and embedded cells. Ultramicroscopy 106:320-325

35. Kodama T, Ohtani H, Arakawa H, Ikai A (2004) Development of confocal laser scanning microscope/atomic force microscope system for force curve measurement. Jpn J Appl Phys 43 (7B):4580-4583

36. Noy A, Huser TR (2003) Combined force and photonic probe microscope with single molecule sensitivity. Rev Sci Instrum $74: 1217-1221$

37. Kim DC, Kim R, Kim HJ, Kim J, Park DH, Kim HS, Joo J (2007) Raman study of polymer-metal hybrid nanotubes using atomic force/confocal combined microscope. Jpn J Appl Phys 46 (8B):5556-5559

38. Anderson MS, Pike WT (2002) A Raman-atomic force microscope for apertureless-near-field spectroscopy and optical trapping. Rev Sci Instrum 73:1198-1203

39. Ichimura $T$, Hayazawa $N$, Hashimoto $M$, Inouye $Y$, Kawata $S$ (2004) Application of tip-enhanced microscopy for nonlinear Raman spectroscopy. Appl Phys Lett 84:1768-1770

40. Ichimura $\mathrm{T}$, Hayazawa $\mathrm{N}$, Hashimoto $\mathrm{M}$, Inouye $\mathrm{Y}$, Kawata $\mathrm{S}$ (2004) Tip-enhanced coherent anti-Stokes Raman scattering for vibrational nanoimaging. Phys Rev Lett 92:220801, (4pp) 
41. Kellermayer MSZ, Karsai Á, Kengyel A, Nagy A, Bianco P, Huber T, Kulcsár Á, Niedetzky C, Proksch R, Grama L (2006) Spatially and temporally synchronized atomic force and total internal reflection fluorescence microscopy for imaging and manipulating cells and biomolecules. Biophys J 91:2665-2677

42. Peng L, Stephens BJ, Bonin K, Cubicciotti R, Guthold M (2007) A combined atomic force/fluorescence microscopy technique to select aptamers in a single cycle from a small pool of random oligonucleotides. Microsc Res Tech 70:372-381

43. Yamada T, Afrin R, Arakawa H, Ikai A (2004) High sensitivity detection of protein molecules picked up on a probe of atomic force microscope based on the fluorescence detection by a total internal reflection fluorescence microscope. FEBS Lett 569:59-64

44. Aydinli A, Serpengüzel A, Vardar D (1996) Visible photoluminescence from low temperature deposited hydrogenerated amorphous silicon nitride. Solid State Commun 98:273-277

45. Lulevich V, Honig C, Ducker WA (2005) An atomic force microscope tip as a light source. Rev Sci Instrum 76:123704, (5pp)

46. Wolford DJ, Scoot BA, Reimer JA, Bradley JA (1983) Efficient visible luminescence from hydrogenated amorphous silicon. Physica B 117-118:920-922

47. Kitazawa M, Shiotani K, Toda A (2003) Batch fabrication of sharpened silicon nitride tips. Jpn J Appl Phys 42(Pt. 1):48444847

48. Fukuma T, Jarvis SP (2006) Development of liquid-environment frequency modulation atomic force microscope with low noise deflection sensor for cantilevers of various dimensions. Rev Sci Instrum 77:043701, (8 pp)

49. Schitter G, Stemmer A (2004) Identification and open-loop tracking control of a piezoelectric tube scanner for high-speed scanning-probe microscopy. IEEE Trans Control Systems Technol $12: 449-454$

50. Zou Q, Leang KK, Sadoun E, Reed MJ, Devasia S (2004) Control issues in high-speed AFM for biological applications: collagen imaging example. Asian J Control 6:164-178

51. Morita S, Yamada H, Ando T (2007) Japan AFM roadmap 2006. Nanotechnol 18:084001, (10 pp)

52. Schiener J, Witt S, Stark M, Guckenberger R (2004) Stabilized atomic force microscopy imaging in liquids using second harmonic of cantilever motion for setpoint control. Rev Sci Instrum 75:2564-2568

53. Uchihashi T, Ando T, Yamashita H (2006) Fast phase imaging in liquids using a rapid scan atomic force microscope. Appl Phys Lett 89:213112, (3 pp)

54. Stark M, Guckenberger R (1999) Fast low-cost phase detection setup for tapping-mode atomic force microscopy. Rev Sci Instrum 70:3614-3619

55. Fantner GE, Hegarty P, Kindt JH, Schitter G, Cidade GAG, Hansma PK (2005) Data acquisition system for high speed atomic force microscopy. Rev Sci Insturm 76:026118, (4pp)

56. Fantner GE, Schitter G, Kindt JH, Ivanov T, Ivanova K, Patel R, Holten-Andersen N, Adams J, Thuner PJ, Rangelow IW, Hansma PK (2006) Components for high-speed atomic fore microscopy. Ultramicroscopy 106:881-887

57. Humphris ADL, Hobbs JK, Miles MJ (2003) Ultrahigh-speed scanning near-field optical microscopy capable of over 100 frames per second. Appl Phys Lett 83:6-8

58. Humphris ADL, Miles MJ, Hobbs JK (2005) A mechanical microscope: high-speed atomic force microscopy. Appl Phys Lett 86:034196, (3pp)

59. Seo Y, Park JH, Moon JB, Jhe W (2000) Fast-scanning shearforce microscopy using a high-frequency dithering probe. Appl Phys Lett 77:4274-4276

60. Seo Y, Jhe W (2002) High-speed near-field scanning optical microscopy with a quartz crystal resonator. Rev Sci Instrum 73:2057-2059
61. Kindt JH, Fantner GE, Cutroni JA, Hansma PK (2004) Rigid design of fast scanning probe microscopes using finite element analysis. Ultramicroscopy 100:259-265

62. Hansma PK, Schitter G, Fantner GE, Prater C (2006) High-speed atomic force microscopy. Science 314:601-602

63. Burgess SA, Walker ML, Sakakibara H, Knight PJ, Oiwa K (2003) Dynein structure and power stroke. Nature 421:715-718

64. Yildiz A, Forkey JN, McKinney SA, Ha T, Goldman YE, Selvin PR (2003) Myosin V walks hand-over-hand: single fluorophore imaging with $1.5 \mathrm{~nm}$ localization. Science 300:2061-2065

65. Forkey JN, Quinlan ME, Shaw MA, Corrie JET, Goldman YE (2003) Three-dimensional structural dynamics of myosin V by single-molecule fluorescence polarization. Nature 422:399-404

66. Warshaw DM, Kennedy GG, Work SS, Krementsova EB, Beck S, Trybus KM (2005) Differential labeling of myosin V heads with quantum dots allows direct visualization of hand-over-hand processivity. Biophys J 88:L30-L32

67. Syed S, Snyder GE, Franzini-Armstrong C, Selvin PR, Goldman YE (2006) Adaptability of myosin V studied by simultaneous detection of position and orientation. EMBO J 25:1795-1803

68. Burgess S, Walker M, Wang F, Sellers JR, White HD, Knight PJ, Trinick J (2002) The prepower stroke conformation of myosin V. J Cell Biol 159:983-991

69. Braig K, Otwinowski Z, Hegde R, Boisvert DC, Joachimiak A, Horwich AL, Sigler PB (1994) The crystal structure of the bacterial chaperonin GroEL at $2.8 \AA$. Nature 371:578-586

70. Xu Z, Horwich AL, Sigler PB (1997) The crystal structure of the asymmetric GroEL-GroES-(ADP) 7 chaperonin complex. Nature 388:741-750

71. Burston SG, Ranson NA, Clarke AR (1995) The origins and consequences of asymmetry in the chaperonin reaction cycle. $\mathrm{J}$ Mol Biol 249:138-152

72. Yifrach O, Horovitz A (1995) Nested cooperativity in the ATPase activity of the oligomeric chaperonin GroEL. Biochemistry 34:5303-5308

73. Lorimer GH (1997) Protein folding. Folding with a two-stroke motor. Nature 388:720-723

74. Rye HS, Burston SG, Fenton WA, Beechem JM, Xu Z, Sigler PB, Horwich AL (1997) Distinct actions of cis and trans ATP within the double ring of the chaperonin GroEL. Nature 388:792-798

75. Rye HS, Roseman AM, Chen S, Furtak K, Fenton WA, Saibil HR, Horwich AL (1999) GroEL-GroES cycling: ATP and non-native polypeptide direct alternation of folding-active rings. Cell 97:325338

76. Azem A, Kessel M, Goloubinoff P (1994) Characterization of a functional $\mathrm{GroEL}_{14}\left(\mathrm{GroES}_{7}\right)_{2}$ chaperonin hetero-oligomer. Science 265:653-656

77. Schmidt M, Rutkat K, Rachel R, Pfeifer G, Jaenicke R, Viitanen P, Lorimer G, Buchner J (1994) Symmetric complexes of GroE chaperonins as part of the functional cycle. Science 265:656-659

78. Engel A, Hayer-Hartl MK, Goldie KN, Pfeifer G, Hegerl R, Muller S, da Silva AC, Baumeister W, Hartl FU (1995) Functional significance of symmetrical versus asymmetrical GroEL-GroES chaperonin complexes. Science 269:832-836

79. Hayer-Hartl MK, Martin J, Hartl FU (1995) Asymmetrical interaction of GroEL and GroES in the ATPase cycle of assisted protein folding. Science 269:836-841

80. Grallert H, Buchner J (2001) Review: a structural view of the GroE chaperone cycle. J Struct Biol 135:95-103

81. Taguchi H, Ueno T, Tadakuma H, Yoshida M, Funatsu T (2001) Single-molecule observation of protein-protein interactions in the chaperonin system. Nature Biotechnol 19:861-865

82. Yokokawa M, Wada C, Ando T, Sakai N, Yagi A, Yoshimura SH, Takeyasu K (2006) Fast-scanning atomic force microscopy reveals the ATP/ADP-dependent conformational changes of GroEL. EMBO J 25:4567-4576 
83. Kobayashi M, Sumitomo K, Torimitsu K (2007) Real-time imaging of DNA-streptavidin complex formation in solution using a high-speed atomic force microscope. Ultramicroscopy 107:184-190

84. Yokokawa M, Yoshimura SH, Naito Y, Ando T, Yagi A, Sakai N, Takeyasu K (2006) Fast-scanning atomic force microscopy reveals the molecular mechanism of DNA cleavage by ApaI endonuclease. IEE Proc Nanobiotechnol 153:60-66

85. Crampton N, Yokokawa M, Dryden DTF, Edwardson JM, Rao DN, Takeyasu K, Yoshimura SH, Henderson RM (2007) Fast-scan atomic force microscopy reveals that the type III restriction enzyme EcoP15I is capable of DNA translocation and looping. Proc Natl Acad Sci USA 104:12755-12760

86. El Kirat K, Burton I, Dupres V, Dufrene YF (2005) Sample preparation procedures for biological atomic force microscopy. $\mathrm{J}$ Microscopy 218:199-207

87. Zhang SF, Rolfe P, Wright G, Lian W, Milling AJ, Tanaka S, Ishihara K (1998) Physical and biological properties of compound membranes incorporating a copolymer with a phosphorylcholine head group. Biomat 19:691-700

88. Vadgama P (2005) Surface biocompatibility. Annu Rep Prog Chem Sect C: Phys Chem 101:14-52

89. Sackmann E (1996) Supported membranes: scientific and practical applications. Science 271:43-48
90. Reviakine I, Brisson A (2000) Formation of supported phospholipid bilayers from unilamellar vesicles investigated by atomic force microscopy. Langmuir 16:1806-1815

91. Scheuring S, Müller DJ, Ringler P, Heymann JB, Engel A (1999) Imaging streptavidin 2D crystals on biotinylated lipid monolayers at high resolution with the atomic force microscope. J Microscopy 193:28-35

92. Reviakine I, Brisson A (2001) Streptavidin 2D crystals on supported phospholipid bilayers: toward constructing anchored phospholipid bilayers. Langmuir 17:8293-8299

93. Stark M, Stark RW, Heckl WM, Guckenberger R (2002) Inverting dynamic force microscopy: From signals to time-resolved interaction forces. Proc Natl Acad Sci USA 99:8473-8478

94. Shekhawat GS, Dravid VP (2005) Nanoscale imaging of buried structures via scanning near-field ultrasound holography. Science 310:89-92

95. Hansma PK, Drake B, Marti O, Gould SAC, Prater CB (1989) The scanning ion-conductance microscope. Science 243:641-643

96. Fukuma T, Kobayashi K, Matsushige K, Yamada H (2005) True molecular resolution in liquid by frequency-modulation atomic force microscopy. Appl Phys Lett 86:193108, (3 pp)

97. Fukuma T, Higgins MJ, Jarvis SP (2007) Direct imaging of lipid-ion network formation under physiological conditions by frequency modulation atomic force microscopy. Pys Rev Lett 98:106101, (4 pp) 